\title{
Neuropsychiatric aspects of the space missions: scientific overview of the last 15 years
}

\begin{abstract}
Since the first spacecraft mission, space medicine has developed and improved techniques that allowed the treatment and prevention of diseases related to the space environment. An example of this were the screening methods created to exclude astronauts who have high chances of developing health problems during the mission. In addition, the human being in the remote and austere space environment das contraindicated if he had some preexisting pathologies such as coronary heart disease, kidney stones, asthma and epilepsy. Furthermore, the selection of astronauts also takes into account their mental health, with previous psychometric and performance evaluation, in addition to the diagnosis of possible psychiatric pathologies.
\end{abstract}

Keywords: behavioral health, neurology, psychiatry, space mission

\author{
Volume 6 Issue I - 202 I \\ Carlos Eduardo Ximenes da Cunha,' Ariadne \\ Figueiredo Oliveira,' Guilherme Fernandes \\ Góis Dantas,' Laís Rytholz Castro,' João \\ Vitor de Omena Jucá,' Gabriela César Falcão \\ Vieira, ${ }^{2}$ Marina Maria Gonzaga Moreira,' \\ Aline dos Santos Carvalho, ${ }^{3}$ Marina Viegas \\ Moura Rezende Ribeiro ${ }^{4}$ \\ 'Medical Student at Centro Universitário Tiradentes Maceió- \\ Alagoas, Brazil \\ ${ }^{2}$ Physician at Faculdade de Medicina Nova Esperança Paraíba, \\ Brazil \\ ${ }^{3}$ Master in Health Sciences, Professor at Centro Universitário \\ Tiradentes Maceió-Alagoas; Specialist in Neurology, Brazil \\ ${ }^{4} \mathrm{PhD}$ in Health Sciences, Professor at Centro Universitário \\ Tiradentes Maceió-Alagoas; Specialist in Ophthalmology, Brazil
}

Correspondence: Carlos Eduardo Ximenes da Cunha, Medical Student at Centro Universitário Tiradentes Maceió-Alagoas, Tel (79)999187437, Email ocaduximenes@gmail.com

Received: January 04, 202I | Published: January 20, 202

\section{Introduction}

Since man's first visit to space, space medicine has developed and improved techniques that have enabled the treatment and prevention of diseases related to the extraplanetary environment. ${ }^{1,2}$ Among the preventive measures, screening methods have been developed with the aim of excluding astronauts with the highest chances of developing health problems during space missions ${ }^{1,3,4}$ Added to this action, it was contraindicated the permanence of the human in the remote and austere space environment if he had pre-existing pathologies such as coronary disease, renal lithiasis, asthma and epilepsy. ${ }^{5}$ Furthermore, the selection of astronauts also considers their mental health, with previous psychometric and performance evaluation, besides the diagnosis of possible psychiatric pathologies..$^{1,6}$

Despite the development of these techniques in order to seek individuals more adapted to the space environment, long-term missions (longer than 6 months) still bring with them factors that affect the mental health of astronauts, as currently observed on the International Space Station (ISS) ${ }^{6,7}$ Among the neuropsychic changes we can cite social isolation, changes in the circadian cycle, distance from home, exposure to radiation, loss of privacy and limited access to medical care. ${ }^{1,7}$

Considering the repercussions of space travel on the mental health of crew members, the National Aeronautics and Space Administration's (NASA) Human Research Program (HRP) denotes the psychic aggravating factors in the extraplanetary environment and divides them into two groups: psychological and interpersonal factors. ${ }^{8}$ The psychological sphere includes asthenia, loss of temporal sense, sleep-related problems, psychosomatic and psychiatric symptoms. ${ }^{8}$ In the interpersonal field we can cite tension between crew members and deprivation of interpersonal relationships. ${ }^{8}$

In addiction, several studies have been carried out in order to establish the relationship between long-term space mission and neuropsychiatric disorders in astronauts. ${ }^{7}$ This work aims to identify and explain the main neuropsychiatric repercussions of human stay in the space environment published in medical literature over the past 15 years.

\section{Methodology}

It is a bibliographic study of the narrative review type used the following stages: recognition of the theme and formation of the research question; definition of inclusion and exclusion criteria for sampling; categorization of studies; evaluation of studies included in the review; discussion of results and synthesis of knowledge.

The main question of the research was the established influence between stressing factors in long-term space missions and the emergence of neuropsychic alterations in astronauts. The object of the analysis consisted of works published in journals indexed in the Latin American and Caribbean Literature in Health Sciences (LILACS), Scientific Electronic Library Online (SciELO) and United States National Library of Medicine (PubMed) databases.

The search occurred between the months of November and December 2020, using the descriptors: behavioral health; space medicine; and space mission, using AND, we established the following relationship: "behavioral health, space medicine AND space mission". We used language filters (texts in Portuguese and 
English), and time for the elaboration of the review, being considered works published in the last 15 years.

In all, 72 scientific articles were screened, all of them in the PubMed database. Subsequently, the titles and abstracts of the papers were independently evaluated by two researchers, taking into consideration the following inclusion criteria: articles available on the Internet; articles published in English; articles published in Portuguese; articles indexed in the Latin American and Caribbean Literature in Scientific, Electronic Library Online (SciELO), United States National Library of Medicine (PubMed) databases and papers published in the last 15 years , from January 2005 to December 2020

The exclusion criteria were: thesis, monograph, non-scientific resources, articles whose full texts were not available and texts that after reading the abstracts did not present significant content on the proposed topic. After evaluating the titles and abstracts, 35 papers were selected, 28 of which were included for discussion after applying the established exclusion criteria.

\section{Results}

After reading and analysis of the 28 studies selected for discussion, taken from the Pubmed and Scielo databases, comprising research over the last 15 years, the main changes caused by the extraplanetary environment in the neuropsychic aspect of the crew members were briefly described. To contemplate our findings, Table 1 summarizes the data found being composed of: authors and date; country of study and the study summary.

Table I Summary of studies used in the review

\begin{tabular}{lll}
\hline Authors and year & Country of the study & Study's abstract \\
\hline Fiedler ER, Carpenter FE. ${ }^{10}$ & United States of America & $\begin{array}{l}\text { Recognition of the importance of psychological analysis in the selection of } \\
\text { candidates, with a focus on improving behavioral health and performance. }\end{array}$ \\
Johnston SL, et al. ${ }^{\prime \prime}$ & United States of America & $\begin{array}{l}\text { The screening of aspiring astronauts involving a long process of medical } \\
\text { evaluation. The study identifies the health conditions that most disqualified } \\
\text { candidates in the Shuttle Era. }\end{array}$
\end{tabular}

Landon LB, Barrett JD, Slack KJ. ${ }^{12} \quad$ United States of America

Fl.ynn CF. ${ }^{13}$

United States of America

Brady JV, et al. ${ }^{14}$

United States of America

Harrison AA. ${ }^{15}$

United States of America

Liang, $\mathrm{F}$ et al. ${ }^{16}$

United States of America

Sturt J. ${ }^{17}$

United States of America

Lugg DJ. ${ }^{18}$

United States of America

Basner M, et al. ${ }^{19}$

United States of America

Guo. et al. ${ }^{21}$

United States of America

Mallis, MM e DeRoshia CW. ${ }^{22}$

United States of America

Suedfeld P. ${ }^{24}$

United States of America

Britt TW, et al. ${ }^{25}$

United States of America

Doarn, Polk e Shepanek. ${ }^{26}$
Ability to give and follow orders, communication, self-care, team care, teamwork and small group living skills are requirements of the crew's expeditionary skills.

The success of a space mission is related to the performance of astronauts, which can be affected by: psychological adaptation to the space environment, deregulation of the circadian cycle and social distancing.

The difficulty in adapting to the space results mainly from confinement, restriction of quality and variety of food, quality of life support systems, high levels of noise, presence of unpleasant odors, loss of privacy and significant decrease in social interactions.

Interpersonal problems between astronauts; sleep dysregulation and circadian cycle; failure in psychosocial adaptation to space and neuropsychic dysfunctions as factors that depress the performance of astronauts during space missions.

Epigenetic factors, such as DNA methylation, are related to the way astronauts adapt to space.

Earth expeditions, even with significant technological and environmenta differences, allow the study of behavioral health in situations of confinement and social isolation, as in long-term space missions.

Similar simulation studies on Earth are used to predict events related to the behavioral health of astronauts during long-term space missions.

The study of six crew members confined for 520 days in a five hundred cubic meter simulator chamber resulted in different responses to isolation and confinement, with depressive symptoms, physical and mental stress and disorders in the circadian cycle being reported.

Misalignment of the circadian cycle and sleep affecting the decreased ability to perform tasks efficiently and health risks.

Sleep loss and dysregulation of the circadian cycle are responsible for decreasing the astronaut's performance, in addition to increasing the chances of accidents occurring.

Salutogenesis, studied in the field of positive psychology, is seen as a device for better adaptation of astronauts to the stressors of space travel.

Astronauts with significant work abilities are better suited to stressors in the space environment.

Physical and mental exhaustion due to physical distance from family, social and Earth resources, as well as difficulty in synchronous communication. 
Table Continued...

\begin{tabular}{|c|c|c|}
\hline Authors and year & Country of the study & Study's abstract \\
\hline Mark S, et al. ${ }^{27}$ & United States of America & $\begin{array}{l}\text { Studies indicate few differences in the behavioral sphere between men and } \\
\text { women due to the rigid process of psychological screening and astronauts' } \\
\text { selection. }\end{array}$ \\
\hline Goel N, et al. ${ }^{28}$ & United States of America & $\begin{array}{l}\text { The study identifies performance differences related to gender of astronauts } \\
\text { during space missions. }\end{array}$ \\
\hline Kiffer, Boerma e Allen. ${ }^{29}$ & United States of America & $\begin{array}{l}\text { Exposure to } 4 \text { Gy of radiation is responsible for oxidative stress in tissues, } \\
\text { mainly affecting the prefrontal cortex and hippocampus. }\end{array}$ \\
\hline Allen AL, et al. ${ }^{30}$ & United States of America & $\begin{array}{l}\text { Rats irradiated with } 0.5 \mathrm{~Gy} 56 \mathrm{Fe} \text { showed changes in density and dendritic } \\
\text { architecture in the hippocampal region. }\end{array}$ \\
\hline Zanni G, et al. ${ }^{3 \prime}$ & United States of America & $\begin{array}{l}\text { Radiation is responsible for decreasing neurogenesis in the hippocampus } \\
\text { region, a brain structure related to memory. }\end{array}$ \\
\hline Perez et al. ${ }^{32}$ & United States of America & $\begin{array}{l}\text { Exposure to galactic radiation raised the level of anxiety after day } 600 \text { in the } \\
\text { rat's experiment. }\end{array}$ \\
\hline Raber et al. ${ }^{33}$ & United States of America & Cognitive changes induced by $56 \mathrm{Fe}$ involve changes in the hippocampal region. \\
\hline SweetT et al. ${ }^{34}$ & United States of America & $\begin{array}{l}\text { Rats irradiated with daily doses of } 0.2 \text { Gy showed an increase in anxiety and } \\
\text { stress levels on day } 600 \text { of the experiment. }\end{array}$ \\
\hline Impey et al. ${ }^{35}$ & United States of America & $\begin{array}{l}\text { The irradiation by } 56 \mathrm{Fe} \text { iron ions led to a dose-dependent alteration in the } \\
\text { hippocampal region, causing cognitive and object recognition deficits. }\end{array}$ \\
\hline Whoolery CW, et al. ${ }^{36}$ & United States of America & $\begin{array}{l}\text { Exposure to } 56 \mathrm{Fe} \text { radiation reduces neurogenesis in the dentate gyrus of the } \\
\text { hippocampus and decreases hippocampal function. }\end{array}$ \\
\hline Albi et al. ${ }^{38}$ & United States of America & $\begin{array}{l}\text { Microgravity can lead to structural thyroid changes that can influence the } \\
\text { dysregulation of the circadian cycle. }\end{array}$ \\
\hline Clément et al. ${ }^{39}$ & United States of America & $\begin{array}{l}\text { Alteration of cortical electrical activity due to microgravity and stressful } \\
\text { situations. }\end{array}$ \\
\hline Boice JD. ${ }^{40}$ & United States of America & $\begin{array}{l}\text { Hight-LET particle irradiation can lead to dementia, Parkinson's disease and } \\
\text { motor neuron. }\end{array}$ \\
\hline
\end{tabular}

Considering the total number of articles included in the systematic review, 25 were from the United States, 2 from China, 1 from Italy, which indicates that the USA concentrates most of the studies and that no research on the subject has been identified in Brazil. The main neuropsychiatric disorders found were those related to sleep disturbance and the circadian cycle; social distancing and the earthly environment; lack of psychological adaptation to the spatial and work environment; as well as the influence of microgravity and radiation. Thus, eight literature addresses the alteration of the mental health of the crew as a result of the factors exposed above.

Three studies have also observed the importance of screening as a method of choice for individuals most suited to long-term space missions, such as a study affirming the epigenetic influence on this ability. In addition, one article brought the difference in performance between sex, on the other hand, another states that, due to pre-selection, this inequality becomes tiny. Finally, nine studies relate radiation to the involvement of the hippocampal region and, consequently, to the neuropsychic alteration and two associate this as a result of microgravity.

\section{Discussion}

The human body faces a series of challenges in the extraplanetary environment to maintain organic homeostasis. ${ }^{5,2,6}$ Thus, the neurological and psychic component of astronauts are greatly affected during longterm space missions. ${ }^{5,2,6}$ In this sense, the Human Research Program (HRP) of the National Aeronautics and Space Administration (NASA) was created with the purpose of understanding the neuropsychics phenomena in astronauts during long-term space travel, considering that the mental health of the crew has a direct relationship with the success of the space mission (Figure 1). ${ }^{9}$

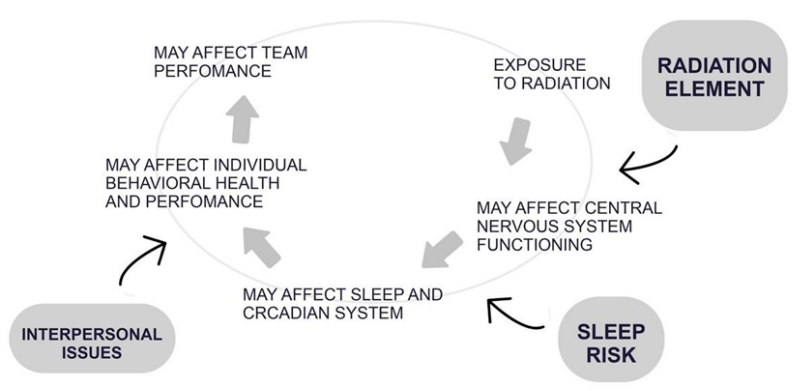

Figure I Stress factors related to behavioral changes in astronauts during space missions.

Author: Cunha, C.E.X. (2020) main stress factors related to changes in the mental health of astronauts. adapted from nasa's human researsh program.

In this perspective, since the first American space program, the Mercury project, the mental health of the candidates influenced the choice of future astronauts. ${ }^{10,11}$ Thus, candidates diagnosed with psychiatric pathologies, such as depression and schizophrenia, a history of trauma or hereditary psychological problems had less chance of being selected. ${ }^{12}$

In addition, for the scientific community, long-term space flights bring the need to understand the process of adaptation of the astronaut to the extraplanetary environment, which can result in significant behavioral and cognitive changes. ${ }^{13,14}$ Yet, studies address four factors that decrease crew's performance, namely: interpersonal problems among astronauts; sleep dysregulation and circadian cycle; fail to psychosocial adaptation in space and neuropsychic dysfunctions (Figure 2). ${ }^{15,13,9,16}$ 


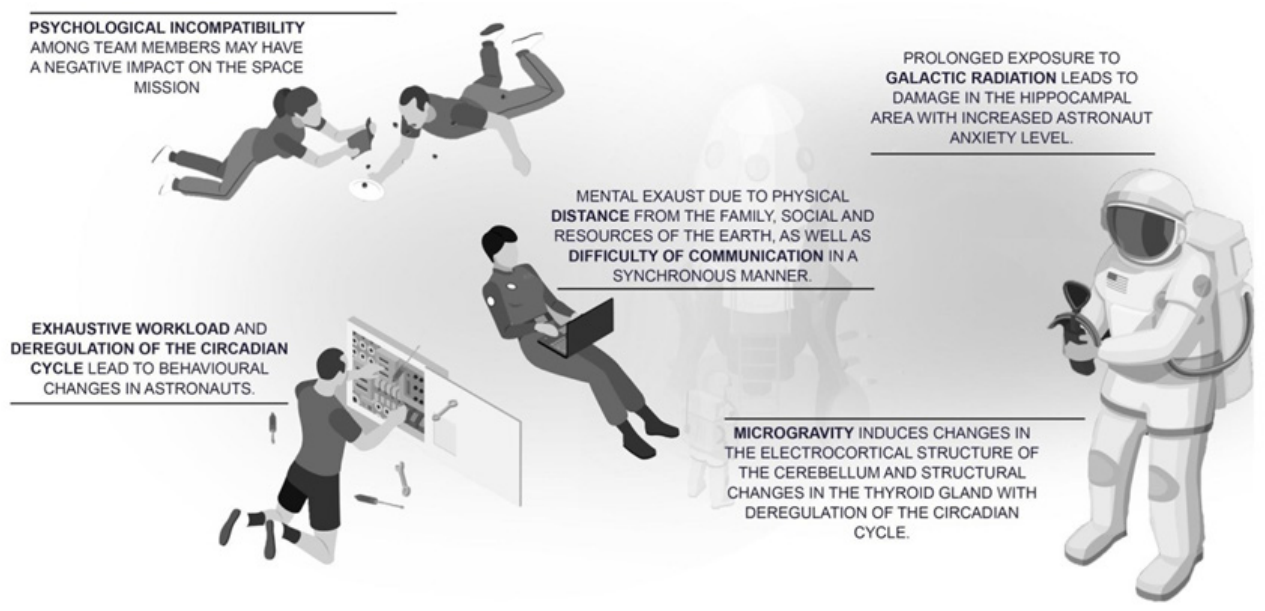

Figure 2 Main neuropsychiatric and behavioral changes in the extraplanetary environment.

Author: Cunha, C.E.X. (2020) (This figure was designed using resources from Freepik.com (https://br.freepik.com/)) changes in neuropsychic spheres reported in this review.

\section{Interpersonal problems}

The interpersonal relationship of the crew, seen through the effectiveness in communication, coordination and cooperation, is directly connected to the success of the space mission..$^{12}$ In this way, the training of crews in space travel has attracted the attention of NASA's Behavioral Health and Performance (BHP). ${ }^{12}$ Therefore, after being selected, aspiring astronauts are submitted to trainings that explore their ability to work as a team and live in small groups. ${ }^{12}$

In this perspective, the current minimum requirements expected of an astronaut must be framed within the expeditionary skills of the crew, including: ability to give and follow orders, communication, self-care, team care, teamwork and living skills in small groups. ${ }^{12}$ Considering that the next trips to the moon and mars will last approximately one to three years, studies with humans have been carried out on earth in order to simulate behavioral responses in a social isolation scenario, confinement and coexistence in group. ${ }^{17,18}$

As an example of personal relationships' importance, Basner et al., 2014 (19) conducted a study with six crew members of different nationalities living for 520 days in a five hundred cubic meter simulator chamber in order to identify neurological and behavioral changes in the participants. ${ }^{19}$ At the end of the experiment, the crew showed different responses to isolation and confinement, with depressive symptoms, physical and mental stress and disturbances in the circadian cycle being reported. ${ }^{19}$ In addition, conflicts between the crew and the mission control were reported as five times more recurrent than those among the study participants themselves. ${ }^{19}$

\section{Sleep deregulation and circadian cycle}

The dysregulation of sleep and the circadian cycle is known as one of the main influencers in neuropsychic alterations, considering that the circadian clock regulates several functions of the organism, such as brain electrical activity, sleep and wake cycle, body metabolism, hormone secretion, body temperature as well as cognition and performance control. ${ }^{20,21,16}$ Thus, when the individual is exposed to the extraplanetary environment, there is a dysfunction of this regulation caused by the high stress level, high luminosity, constant noise, irregular working hours and lack of comfort. ${ }^{21,13}$ The changes associated involve reduced concentration, mood changes, malaise, performance errors, decreased social interaction leading to inefficient communication and even space accidents. ${ }^{13,22}$

That said, the inadequate rest of crew's members is described by Laura K. Barger, who states in her research with 64 astronauts that sleep disturbance comes well before the crew starts the mission (about 3 months earlier) and that the use of sleeping pills was widespread among members. ${ }^{23}$ Therefore, NASA, through HRP, pointed out the changes in the circadian cycle, the few hours of rest and fatigue as some of the elements that can cause a reduction in the astronauts' performance. ${ }^{9}$

\section{Psychosocial adaptation to space}

Within the scope of psychosocial adaptation, living for months or years in space remains a challenge for astronauts, considering the expressive differences between the earth's and extra-planetary environments. ${ }^{14}$ The difficulty of adaptation to space results mainly from confinement, from the restriction of quality and variety of food, from the quality of life support systems and the high levels of noise, from the presence of unpleasant odors, from the loss of privacy and the significant decrease in social interactions. ${ }^{14,24,25}$

In addiction, the deprivation of synchronously communication with the Earth, the physical distance both from our planet and from family and social environments, the need for great concentration and excessive concerns are considered points with the greatest psychological impact in crew, as well as cultural misunderstanding. ${ }^{26,13}$

Other factors related to behavioral adaptation in the extraplanetary environment are connected with astronauts' gender and epigenetic issues. ${ }^{27,28,16}$ The most recent studies indicate little differences in the behavioral sphere between men and women, even though females have twice as much chances of developing anxiety and depression. According to Mark S. et al., 2014 (27) this similarity of risk is due to the rigid process of psychological screening and selection of astronauts. ${ }^{27}$

In regard to epigenetics, a study made by Liang et al., 2019 (16) signaled a possible phenotypic relationship between changes in the glucose cycle and mood changes in astronauts during a simulated space mission. ${ }^{16}$ In the experiment, it was observed that the methylation profile of cellular DNA, different between individuals, was remodeled as the mood changes in the participants. ${ }^{16}$ 


\section{Neuropsychic dysfunction}

Besides the conditions related to neuropsychic changes, an important problem is the prolonged exposure to cosmic radiation from celestial bodies in the milk pathway. ${ }^{29,30}$ This is because the rate of radiation absorbed by the body is considerably higher in interplanetary mission, that is, from one celestial body to another. In addition, the maximum time man has ever been exposed to high levels of ionizing energy was in the Apollo program, with a maximum duration of 13 days. ${ }^{29}$ In this context, the relationship between the dose of galactic radiation absorbed by the body and the emergence of cognitive and behavioral changes has been the subject of several studies, since the next manned mission to Mars will last approximately 800-1000 days, being almost half of this time spent on the surface of the planet with an average radiation rate of $0.481 \pm 0.080 \mathrm{mGy} /$ day. $^{29,31}$

A study by Perez et al.,2020 (32) simulated the extraplanetary environment with chronic and low intensity exposure of galactic radiation (0.1-0.4 Gy/day) with rats. ${ }^{32}$ At the time, it was observed that the rats did not present significant changes in the cognitive sphere, however, they had their level of anxiety increased around day 600 of the experiment. . $^{32,31,30}$ The pathophysiological explanation for these changes resulting from radiation is the presence of ionizing particles with high energetic value as responsible for oxidative stress in human tissues, being the prefrontal cortex and hippocampus regions of the central nervous system that present neuro inflammation and astrogliosis in the face of exposure to $4 \mathrm{~Gy}^{29,33,34}$

Iron ion irradiation $\mathrm{Fe}$ causes synaptic changes in neuronal networks related to plasticity and neuronal memory with epigenetic changes in cytosine methylation $(5 \mathrm{mC})$ and hydroxymethyllation $(5 \mathrm{hmC})$, which affect the stability of the hippocampal work, neuronal structure related to cognitive function. ${ }^{35,36,34,31}$

Finally, a point to be observed are neurocognitive changes caused directly or indirectly by microgravity, being through electrocortical changes in certain brain structures and by thyroid dysfunction, respectively. ${ }^{37,38}$ In the microgravity, cortical electrical circuits undergo changes resulting from emotional stress in the context of anxiety, monotony, and limited mobility to which astronauts are exposed. ${ }^{39,40}$ Also, the deprivation of gravitational force can lead to cellular alterations in the thyroid, such as hyperactivity of the enzyme caspase-3, apoptosis of thyrocytes and decreased sensitivity of the thyrootropin receptor. ${ }^{38,39}$ Such glandular alterations reflect in important physiological alterations throughout the body, including in the circadian cycle and in the astronauts' mood. ${ }^{38}$

\section{Conclusion}

Interplanetary space travel brings with it the complexity of physiological adaptation of the human body to the microgravity environment, prolonged exposure to radiation and social isolation of astronauts. The central nervous system is directly affected by these factors and behavioral and cognitive changes in astronauts can result in negative outcomes in space missions, such as: dysregulation of sleep and the circadian cycle, increased level of anxiety and alterations in the astronaut's mood.

In view of the current phase of human exploration into space, it is necessary to conduct more studies in the area in order to understand more assertively the neuropsychiatric changes in astronauts in the extraplanetary environment.

\section{Acknowledgments}

None.

\section{Conflicts of interest}

No potential conflict of interest relevant to this article was reported.

\section{References}

1. Martyn Y. Changes in space medicine over the last 50 years. Occup Med (Lond). 2019; 69(5):314-315.

2. Launius, RD. The historical dimension of space exploration: reflections and possibilities. Space Policy. 2000;16(1):23-38.

3. Berry CA, Hoffler GW, Jernigan CA, et al. History of space medicine: the formative years at NASA. Aviatio Space and Environmental Medicine. 2009;80(4);345-352.

4. Cucinotta, Francis Cacao, Eliedonna Kim, et al. Premkumar.Non-targeted effects lead to a paradigm shift in risk assessment for a mission to the earth's moon or martian moon phobos. Radiation Protection Dosimetry. 2018.

5. Hodkinson PD, Anderton RA, Posselt BN, et al. An overview of space medicine. Br J Anaesth. 2017;119(suppl_1):i143-i153.

6. Collins Daniel L. Psychological issues relevant to astronaut selection for long-duration space flight: a review of the literature. Journal of Human Performance in Extreme Environments. 2003;7(1).

7. Morris NP. Behavioral health policy for human spaceflight. Aerosp Med Hum Perform. 2018;89(12):1068-1075.

8. Zhang QJ, Bai YQ. Psychological issues in manned spaceflight. Space Med MedEng (Beijing). 1999;12(2):144-148.

9. McPhee JC, Charles JB. Human health and performance risks of space exploration missions: evidence reviewed by the NASA human research program. houston, texas: national aeronautics and space administration. Risk of Behavioral and Psychiatric Conditions. 2009;1:3-85.

10. Fiedler ER, Carpenter FE. Evolution of the behavioral sciences branch of the space medicine and health care systems office at the johnson space center. Aviat Space Environ Med. 2005;76(6 Suppl):B31-35.

11. Johnston SL, Blue RS, Jennings RT, et al. Astronaut medical selection during the shuttle Era: 1981-2011. Aviation, Space, and Environmental Medicine. 2014;85(8):823-827.

12. Landon LB, Slack KJ, Barrett JD. Teamwork and collaboration in longduration space missions: Going to extremes. American Psychologist. 2018;73(4):563-575.

13. Flynn CF. An operational approach to long-duration mission behavioral health and performance factors. Aviat Space Environ Med. 2005;76(6 Suppl):B42-51.

14. Brady JV. Behavioral health: the propaedeutic requirement. Aviat Space Environ Med. 2005;76(6 Suppl):B13-24.

15. Harrison AA. Behavioral health: integrating research and application in support of exploration missions. Aviat Space Environ Med. 2005;76(6 Suppl):B3-12.

16. Liang F, Lv K, Wang Y, et al. Personalized epigenome remodeling under biochemical and psychological changes during long-term isolation environment. Frontiers in Physiology. 2019;31.

17. Stuster J. Analogue prototypes for lunar and mars exploration. Aviat Space Environ Med. 2005 Jun;76(6 Suppl):B78-83.

18. Lugg DJ. Behavioral health in Antarctica: implications for long-duration space missions. Aviat Space Environ Med. 2005;76(6 Suppl):B74-77.

19. Basner M, Dinges DF, Mollicone DJ, et al. Psychological and behavioral changes during confinement in a 520-Day simulated interplanetary mission to mars. PLoS ONE. 2014;9(3):e93298.

20. Wu B, Wang Y, Wu X, et a.. On-orbit sleep problems of astronauts and countermeasures. Mil Med Res. 2018; 30;5(1):17. 
21. Guo JH, Qu WM, Chen SG. et al. Keeping the right time in space: importance of circadian clock and sleep for physiology and performance of astronauts. Military Med Res. 2014;1:23.

22. Mallis MM, DeRoshia CW. Circadian rhythms, sleep, and performance in space. Aviat Space Environ Med. 2005;76(6 Suppl):B94-107.

23. Barger LK, Flynn-Evans EE, Kubey A, et al. Prevalence of sleep deficiency and use of hypnotic drugs in astronauts before, during, and after spaceflight: an observational study. Lancet Neurol. 2014;13(9):904-912.

24. Suedfeld P. Invulnerability, coping, salutogenesis, integration: four phases of space psychology. Aviat Space Environ Med. 2005;76(6 Suppl):B61-66.

25. Britt TW, Sytine A, Brady A, et al. Enhancing the meaningfulness of work for astronauts on long duration space exploration missions. Aerospace Medicine andHuman Performance. 2017;88(8):779-783.

26. Doarn CR, Polk JD, Shepanek M. Health challenges including behavioral problems in long-duration spaceflight. NeurolIndia. 2019;67(S2):190 195.

27. Mark S, Scott GBI, Donoviel DB, et al. The impact of sex and gender on adaptation to space: executive summary. Journal of Women's Health. 2014;23(11):941-947.

28. Goel N, Bale TL, Epperson CN, et al. Effects of Sex and Gender on Adaptation to Space: Behavioral Health. Journal of Women's Health. 2014;23(11):975-986.

29. Kiffer F, Boerma M, Allen A. Behavioral effects of space radiation: A comprehensive review of animal studies. Life Sciences in Space Research. 2019;21:1-21.

30. Allen AR, Raber J, Chakraborti A, et al. 56Fe irradiation alters spine density and dendritic complexity in the mouse hippocampus. Radiation Research. 2014;184(6):586-594.

31. Zanni G, Deutsch H, Rivera P, et al. Whole-body $12 \mathrm{C}$ irradiation transiently decreases mouse hippocampal dentate gyrus proliferation and immature neuron number, but does not change new neuron survival rate. International Journal of Molecular Sciences. 2018;19(10):3078.
32. Perez RE, Younger S, Bertheau E, et al. Effects of chronic exposure to a mixed field of neutrons and photons on behavioral and cognitive performance in mice. Behavioural Brain Research. 2020;379:112377.

33. Raber Jacob, Allen Anti no R, Weber Sydney, et al. Effect of behavioral testing on spine density of basal dendrites in the CA1 region of the hippocampus modulated by $56 \mathrm{Fe}$ irradiation. Behavioural Brain Research. 2016;302:263-268.

34. Sweet TB, Panda N, Hein AM, et al. Central nervous system effects of whole-body proton irradiation. Radiation Research. 2014;182(1):18.

35. Impey S, Jopson T, Pelz C, et al. Short- and long-term effects of $56 \mathrm{Fe}$ irradiation on cognition and hippocampal DNA methylation and gene expression. BMC Genomics. 2016;17(1).

36. Whoolery CW, Walker AK, Richardson DR, et al. Whole-body exposure to28si-radiation dose-dependently disrupts dentate gyrus neurogenesis and proliferation in the short term and new neuron survival and contextual fear conditioning in the long term. Radiation Research. 2017;188(5):532551.

37. Van Ombergen A, Demertzi A, Tomilovskaya E, et al. The effect of spaceflight and microgravity on the human brain. Journal of Neurology. 2017;264(S1);18-22.

38. Albi E, Krüger M, Hemmersbach R, et al. Impact of gravity on thyroid cells. International Journal of Molecular Sciences. 2017;18(5):972.

39. Clément GR, Boyle RD, George KA, et al. Challenges to the central nervous system during human spaceflight missions to Mars. Journal of Neurophysiology. 2020;123(5):2037-2063

40. John D. Boice Jr. The million person study relevanceto space exploration and mars. International Journal of Radiation Biology. 2019. 